\title{
QUASICONFORMAL MAPPINGS, WITH APPLICATIONS TO DIFFERENTIAL EQUATIONS, FUNCTION THEORY AND TOPOLOGY
}

BY LIPMAN BERS

The theory of quasiconformal mappings is nearly 50 years old (see [44] for references to the papers by Grötzsch, Ahlfors Lavrent'ev and Morrey from the 20's and 30's) and the interest in them does not seem to wane. These mappings may be studied for their own sake or as a tool for attacking other mathematical problems; they are indeed a powerful and flexible tool. The purpose of this lecture is to state two basic theorems about quasiconformal mappings in two dimensions (the existence theorem, the first version of which is due to Gauss, and Teichmüller's theorem about extremal quasiconformal mappings) and to discuss some applications of these theorems. The discussion will necessarily be sketchy and several important topics will be slighted or not even mentioned. (Some of those are covered in [15], [20], [23].)

(At St. Louis I learned about an interesting application, due to J. Sachs and $\mathrm{K}$. Uhlenbeck, to the theory of minimal surfaces. Added in proof.)

Lack of time and of competence prevents me from saying anything about the subtle theory of quasiconformal mappings in $n$-space, initiated in a short note [48] by my late teacher Loewner, and developed by Gehring, Väisälä and others (see the references in [32], [67]), a theory which also has important applications (Mostow [55]).

We begin by defining the concept of quasiconformality.

1. Recall that a Riemannian metric in a domain in the $(x, y)$ plane is defined by a quadratic differential form

$$
d s^{2}=E(x, y) d x^{2}+2 F(x, y) d x d y+G(x, y) d y^{2}
$$

with

$$
E G-F^{2}>0, \quad E>0 .
$$

Setting $x+i y=z, x-i y=\bar{z}$, (1) can be rewritten as

$$
d s^{2}=\Lambda(z)^{2}|d z+\mu(z) d \bar{z}|^{2}
$$

where $\Lambda$ is a real-valued and $\mu$ a complex-valued function. Condition (2) becomes

$$
\Lambda>0, \quad|\mu|<1 .
$$

A mapping $x+i y=z \mapsto w=u+i v$ is called conformal with respect to $d s$ if it preserves orientation and, except at isolated points, takes angles measured by the metric (1) into equal Euclidean angles.

Using the standard notations

$$
\frac{\partial}{\partial z}=\frac{1}{2}\left(\frac{\partial}{\partial x}-i \frac{\partial}{\partial y}\right), \quad \frac{\partial}{\partial \bar{z}}=\frac{1}{2}\left(\frac{\partial}{\partial x}+i \frac{\partial}{\partial y}\right),
$$

the requirement that $w$ be conformal with respect to the metric (1) may be

Retiring Presidential Address delivered before the 83rd Annual Meeting of the American Mathematical Society in St. Louis, Missouri, on January 28, 1977. Work partially supported by the National Science Foundation; received by the editors February 25, 1977. 
written as

$$
\partial w / \partial \bar{z}=\mu(z) \partial w / \partial z .
$$

This is the classical Beltrami equation, which goes over into the CauchyRiemann equations for $u$ and $v(\partial w / \partial \bar{z}=0)$ if $\mu(z) \equiv 0$. Note that $\Lambda$ does not enter into (3), as was to be expected. From now on we call solutions of (3) $\mu$-conformal functions.

(TeCHNICAL REMARK. Solutions of (3) are assumed to be continuous and to have distribution derivatives which are, locally, square integrable functions; equation (3) is assumed to hold almost everywhere.)

A homeomorphism $w$ is called quasiconformal if it is $\mu$-conformal for some $\mu$ with

$$
\|\mu\|_{\infty}=\operatorname{ess} \sup |\mu(z)|<1 .
$$

If so, the number

$$
K(w)=\left(1+\|\mu\|_{\infty}\right) /\left(1-\|\mu\|_{\infty}\right)
$$

is called the dilatation of $w$. If $w$ is a diffeomorphism, $K(w)$ is the supremum of the ratios of the major to the minor axes of infinitesimal ellipses into which $w$ takes infinitesimal circles in the domain considered. A similar definition can always be used, since every quasiconformal homeomorphism is differentiable almost everywhere.

(We have stated the so-called analytic definition of quasiconformality. Concerning the equivalent geometric definition, see [4], [46].)

2. If $\mu(z)$ is a measurable function defined for all $z \in \mathbf{C}$ and satisfying (4), then there exists a unique $\mu$-conformal homeomorphism

$$
z \mapsto w^{\mu}(z)
$$

of $\mathbf{C}$ onto itself satisfying $w^{\mu}(0)=0, w^{\mu}(1)=1$. This $w^{\mu}$ has a modulus of continuity depending only on $\|\mu\|_{\infty}$. (More precisely, it satisfies a Hölder condition

$$
\left\langle w^{\mu}\left(z_{1}\right), w^{\mu}\left(z_{2}\right)\right\rangle \leqslant C\left\langle z_{1}, z_{2}\right\rangle^{\alpha},
$$

where $\langle$,$\rangle denotes spherical distance and the positive constants C, \alpha$ depend only on $\|\mu\|_{\infty}$.)

Also, $w^{\mu}$ is as "nice" as $\mu$ permits it to be. (More precisely, $w^{\mu}$ is real analytic if $\mu$ is, infinitely differentiable if $\mu$ is, has Hölder continuous partial derivatives of order up to $k+1$ if $\mu$ has such derivatives of order up to $k$, has generalized derivatives which are locally $L_{p}$ for some $p=p\left(\|\mu\|_{\infty}\right)>2$ if $\mu$ is merely measurable.)

Finally, $w^{\mu}$ depends "nicely" on $\mu$. (For instance, if $\left\|\mu_{j}\right\|_{\infty} \leqslant k<1$ and $\mu_{j} \rightarrow \mu$ a.e., then $w^{\mu_{j}} \rightarrow w^{\mu}$ uniformly on compact subsets. If $\mu$, considered as an element of an appropriate Banach space, depends holomorphically on some complex parameters, so does $w^{\mu}(z)$.)

If $\mu(z)$ is defined in the upper half-plane $U=[y>0]$, and $\|\mu\|_{\infty}<1$, then there exists a unique homeomorphism

$$
z \mapsto w_{\mu}(z)
$$

of $U$ onto itself, which is continuous on $\mathbf{R} \cup\{\infty\}$ and keeps $0,1, \infty$ fixed. 
This follows from the result about $w^{\mu}$. Indeed,

$$
w_{\mu}=w^{\nu} \mid U \quad \text { where } \nu(\bar{z})=\overline{\nu(z)}, \nu \mid U=\mu .
$$

The mapping $w_{\mu}$ has properties similar to those of $w^{\mu}$, except that the dependence on $\mu$ is real analytic rather than holomorphic.

The existence and uniqueness theorem stated above is the end result of a long development, starting with Gauss who proved a local existence theorem for a real analytic $\mu$, and involving Korn and Lichtenstein, who recognized the importance of Hölder conditions, Lavrent'ev, Morrey who permitted $\mu$ to be discontinuous, Chern, Mori, Vekua, Boyarskii, Ahlfors, Ahlfors and Bers, Earle and Schatz and others; see [5] for reference. The simplest proof of the theorem utilizes the Calderon-Zygmund inequality for the two-dimensional Hilbert transform; it uses no deep function theoretical results [4], [5], [46].

3. Applications of quasiconformal mappings to partial differential equations occur already in the early papers by Lavrent'ev and by Morrey [54], whose approach has been continued by Nirenberg [57], [58]. Here we shall consider a partial differential equation of the form

$$
A_{11} \varphi_{x x}+2 A_{12} \varphi_{x y}+A_{22} \varphi_{y y}=0
$$

where

$$
A_{j k}=A_{j k}\left(x, y, \varphi_{x}, \varphi_{y}, \varphi\right)
$$

(so that the equation is, in general, nonlinear). We assume that for all values of $x, y, \varphi_{x}, \varphi_{y}, \varphi$ considered equation (10) is elliptic, i.e.,

$$
A_{11}>0, \quad A_{11} A_{22}-A_{12}^{2}>0,
$$

and even uniformly elliptic, i.e.,

$$
0<\frac{A_{11}+A_{22}}{\sqrt{A_{11} A_{22}-A_{12}^{2}}} \leqslant M<+\infty .
$$

A trivial example is the Laplace equation $\varphi_{x x}+\varphi_{y y}=0$. Nontrivial examples are the equation of minimal surfaces

$$
\left(1+\varphi_{y}^{2}\right) \varphi_{x x}-2 \varphi_{x} \varphi_{y} \varphi_{x y}+\left(1+\varphi_{x}^{2}\right) \varphi_{y y}=0,
$$

which is uniformly elliptic for $\varphi_{x}^{2}+\varphi_{y}^{2}<$ const, and the potential equation of a compressible flow

$$
\left(c^{2}-\varphi_{x}^{2}\right) \varphi_{x x}-2 c \varphi_{x} \varphi_{y} \varphi_{x y}+\left(c^{2}-\varphi_{y}^{2}\right) \varphi_{y y}=0,
$$

where $c$ is the speed of sound, which is a given function of the flow-speed $\left(\varphi_{x}^{2}+\varphi_{y}^{2}\right)^{1 / 2}$

$$
c^{2}=1-\alpha\left(\varphi_{x}^{2}+\varphi_{y}^{2}\right), \quad \alpha=\text { const. }
$$

Equation (11) is uniformly elliptic as long as the Mach number $M=\sqrt{\varphi_{x}^{2}+\varphi_{y}^{2}} / c$ is bounded away from 1, i.e. for uniformly subsonic flows.

A direct calculation shows that if $\varphi(x, y)$ satisfies (10) in some domain $D$, then the complex gradient $w=\varphi_{x}-i \varphi_{y}$ is $\mu$-conformal, for some $\mu$ depending on $\varphi(x, y)$, but always satisfying 


$$
\|\mu\|_{\infty} \leqslant \kappa(M)<1 .
$$

(Recall that if (10) is the Laplace equation, $w$ is conformal, i.e., holomorphic.)

Now it is almost self-evident that a $\mu$-conformal function is a holomorphic function of any $\mu$-conformal homeomorphism. Hence the complex gradient $w$ satisfies

$$
w=f \circ w^{\mu} \mid D
$$

where $f$ is holomorphic.

Formula (13) has interesting consequences. Since $w^{\mu}$ is a topological mapping and $f$ is holomorphic, $w$ can have only isolated zeros, unless it vanishes identically. It follows that solutions of (10) have the unique continuation property: if $\varphi=0$ in any open set, $\varphi \equiv 0$. (This is true also for elliptic equations of 2 nd order in $n$-space, but requires more continuity assumptions for the coefficients.) Also, since $w^{\mu}$ is a homeomorphism, the level lines of a solution $\varphi$ are topologically the same as the level lines of a harmonic function.

Suppose now we want to solve a boundary value problem for equation (10), say the Dirichlet problem in which the values of $\varphi$ are prescribed on the boundary of the domain $D$. Experts in partial differential equations know that in order to prove the existence of a solution it is usually sufficient to prove a sufficiently strong a priori estimate, i.e., a statement that a solution of the problem, if it exists, satisfies certain inequalities. A priori estimates can be derived from formula (13) since for $w^{\mu}$ we know the modulus of continuity (see (12) and (7)) and $f$ is holomorphic and there are many powerful inequalities for holomorphic functions, for instance, Privaloff's theorem connecting the Hölder continuity of the imaginary part of a holomorphic function with that of the real part.

Indeed, using Privaloff's theorem and a representation of the form (13), I gave, many years ago, a rigorous proof for the existence of a subsonic flow past a given airfoil profile, satisfying the so-called Kutta-Joukowski condition [7]. I mention this result for sentimental reasons: I worked on subsonic flows during World War II, remained interested in them for a long time, and learned about quasiconformal mappings in order to apply them to flow problems.

Later, in two joint papers with L. Nirenberg, the method based on formula (13) has been extended to general, quasilinear second-order elliptic equations, and systems of first order equations, in the plane [21], [22] .

4. We pass to an application of quasiconformal mappings to topology. Let $S$ be a compact differentiable orientable surface of genus $p$ (a sphere with $p$ handles), and let $\Gamma$ be the group of those diffeomorphisms of $S$ onto itself which are homotopic to the identity. With the $C_{\infty}$ topology, $\Gamma$ becomes a topological group. Earle and Eells [25] showed, using quasiconformal mappings, that $\Gamma$ can be continuously retracted onto $S O(3)$ if $p=0$, onto a torus if $p=1$, onto a point if $p>1$. (For the sake of brevity we do not state their results for nonorientable surfaces or surfaces with boundary, cf. Earle and Schatz [28].) The result for $p=0$ was originally proved by Smale [62], by topological methods. Subsequently Gramain [34] obtained a topological proof for the whole theorem. 
I reproduce the proof for $p=0$. The sphere may be identified, by stereographic projection, with $\hat{\mathbf{C}}=\mathbf{C} \cup\{\infty\}$. Every element $f \in \Gamma$ can be written uniquely in the form $f=g \circ w$ where $w$ keeps the points $0,1, \infty$ fixed and $g$ is a Möbius transformation, i.e., a conformal self-mapping of $\mathbf{C}$ described by a unimodular matrix:

$$
g=\left(\begin{array}{ll}
A & B \\
C & D
\end{array}\right), \quad A D-B C=1 \text { or } g(z)=\frac{A z+B}{C z+D} .
$$

Since $w$ is a diffeomorphism, $w=w^{\mu}$ for some infinitely differentiable $\mu$ (with a proper interpretation of this term at $z=\infty$ ). On the other hand, $g$ can be written, uniquely, as

$$
g=\left(\begin{array}{cc}
a & b \\
-\bar{b} & \bar{a}
\end{array}\right)\left(\begin{array}{cc}
r & 0 \\
0 & r^{-1}
\end{array}\right)\left(\begin{array}{cc}
1 & \alpha \\
0 & 1
\end{array}\right) \text { with } r>0
$$

(Iwasawa decomposition). Let $0 \leqslant t \leqslant 1$ and set

$$
g_{t}=\left(\begin{array}{cc}
a & b \\
-\bar{b} & \bar{a}
\end{array}\right)\left(\begin{array}{cc}
r^{t} & 0 \\
0 & r^{-t}
\end{array}\right)\left(\begin{array}{cc}
1 & t \alpha \\
0 & 1
\end{array}\right), \quad w_{t}=w^{t \mu} .
$$

The theorem in $\$ 2$ assures that $f_{t}=g_{t} \circ w_{t} \in \Gamma$ depends continuously on $t$. But $f_{1}=f$ and

$$
f_{0}(z)=(a z+b) /(-\bar{b} z+\bar{a}), \quad|a|^{2}+|b|^{2}=1,
$$

represents, via stereographic projection, a rigid rotation of the unit sphere.

We shall mention the case $p>0$ later (see $\S 10$ ).

5. The most fruitful applications of quasiconformal mappings occur, naturally, in complex function theory, in particular in the theory of Riemann surfaces. A Riemann surface is a one-dimensional complex manifold or, which is the same, an oriented surface on which one has defined a conformal structure, that is a consistent way of measuring angles. We may always think of a Riemann surface as an oriented, sufficiently smooth, surface embedded in Euclidean space, provided we agree that two surfaces are identical qua Riemann surfaces if there is a conformal (topological, orientation and angle preserving) bijection between them.

Let $S$ be a Riemann surface of type $(p, n)$, that is, one obtained from a compact surface of genus $p$ by removing $n$ distinct points. The celebrated uniformization theorem, conjectured by Klein and by Poincaré in 1882 and proved by Poincare and by Koebe in 1907, asserts that if

$$
2 p-2+n>0
$$

then $S$ admits an (essentially unique) representation of the form

$$
S=U / G
$$

where $U=[y>0]$ is the upper half-plane and $G$ a torsion-free Fuchsian group, i.e., a discrete group of real Möbius transformations (conformal self-mappings of $U$ ). The existence theorem in $\$ 2$ yields a short proof of the uniformization theorem [10].

There is a simple geometric method, going back to Poincaré, to construct a Fuchsian group $G_{0}$ such that $U / G_{0}$ is some Riemann surface of the desired type $(p, n)$ : 


$$
U / G_{0}=S_{0} \text {. }
$$

There is a diffeomorphism $f: S_{0} \rightarrow S$, where $S$ is the given surface, and if $d s$ is some Riemannian metric on $S$ which respects the conformal structure of $S$, we can pull it back to $S_{0}$, by $f$, and obtain on $S_{0}$ a smooth Riemannian metric $d s_{0}$ which will, in general, not respect the conformal structure of $S_{0}$. Now (16) means that there is a conformal universal covering $\pi_{0}: U \rightarrow S_{0}$ with covering group $G_{0}$. Using $\pi_{0}$ we pull back $d s_{0}$ to a Riemannian metric on $U$ which we write in the form $\Lambda \mid d z+\mu d \bar{z}$. Thus we obtain in $U$ a function $\mu(z)$, with $\|\mu(z)\|_{\infty}<1$. Next, we let $w_{\mu}$ be the normalized $\mu$-conformal self-mapping of $U$ (cf. \$2) and we form the group

$$
G_{\mu}=w_{\mu} G w_{\mu}^{-1} .
$$

A direct calculation shows that this group is a discrete group of Möbius transformations, mapping $U$ onto itself, thus a Fuchsian group. If we define the map $\pi: U \rightarrow S$ by the commutativity of the diagram

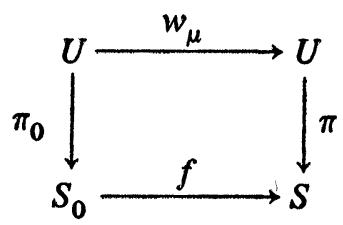

we can verify that $\pi$ is conformal and a universal covering with covering group $G_{\mu}$. Hence $U / G_{\mu}=S$, as required.

The same method yields all classical uniformization theorems for Riemann surfaces of finite type and, which is more important, new uniformization theorems which seem, at the present time, inaccessible to classical methods.

6. Let us begin, as before, with the Fuchsian group $G_{0}$, the Riemann surface $S_{0}=U / G_{0}$ and another Riemann surface $S=f\left(S_{0}\right)$, and let us construct as before the function $\mu(z), z \in U$. However, instead of forming the $\mu$-conformal mapping $w_{\mu}: U \rightarrow U$, let us extend the definition of $\mu$ to all $\mathbf{C}$ by setting

$$
\mu(z)=0 \text { for } z \in L,
$$

where $L=[y<0]$ denotes the lower half-plane. (The horrid singularities of $\mu$ along the real axis are irrelevant.) Then

$$
G^{\mu}=w^{\mu} G_{0}\left(w^{\mu}\right)^{-1}
$$

is again a discrete group of Möbius transformations. In general $w^{\mu}$ will not be symmetric, $w^{\mu}(U)$ will not be $U$ but some Jordan domain $\Delta_{1}$ bounded by the curve

$$
C=w^{\mu}(\mathbf{R}) \cup\{\infty\}
$$

and $w^{\mu}(L)=\Delta_{2}$ will be another Jordan domain. Furthermore, $\Delta_{1} / G^{\mu}$ will be $S$ and $\Delta_{2} / G^{\mu}$ will be $L / \underline{G}_{0}=\bar{S}_{0}$, since $w^{\mu} \mid L$ is now conformal.

If we set $S=S_{1}$ and $\bar{S}_{0}=S_{2}$, and write $G$ for $G^{\mu}$, we obtain the theorem on simultaneous uniformization [9]: Given two Riemann surfaces $S_{1}$ and $S_{2}$ of the same type $(p, n)$, with $2 p-2+n>0$, there exists a (torsion-free) quasi- 
Fuchsian group $G$, i.e., a discrete group of Möbius transformations which leaves a directed Jordan curve $C$ fixed, such that

$$
\Delta_{1} / G=S_{1}, \quad \Delta_{2} / G=S_{2},
$$

$\Delta_{1}$ and $\Delta_{2}$ being the two components of the complement of $C$.

Note that by (17) the group $G$ is canonically isomorphic to the fundamental groups of both surfaces $S_{1}$ and $S_{2}$. Thus $G$ defines two Riemann surfaces and an isomorphism between their fundamental groups. It can be shown that these data determine $G$ uniquely, up to conjugation.

A quasi-Fuchsian group is an example of a Kleinian group, i.e. a discrete group of Möbius transformations which acts properly discontinuously on some open subset of $\hat{\mathbf{C}}$. The " $\mu$-trick" used in the proof above has been applied by Maskit in his penetrating investigations of Kleinian groups which led, among other things, to a clarification and deepening of the classical results of Klein and Koebe, and to a complete classification of important species of Kleinian groups [50], [51], [52].

7. Using the theorem on quasi-Fuchsian groups stated in $\S 6$, P. A. Griffiths [35] obtained a far-reaching generalization of the Poincaré-Klein-Koebe theorem to algebraic varieties (over $\mathbf{C}$ ) of arbitrary dimension. For the sake of brevity we state it for affine rather than for projective varieties.

Let $\boldsymbol{A}$ be an affine algebraic variety, that is, the set in $\mathbf{C}^{N}$ of common zeros of a collection of polynomials in the variables $\left(z_{1}, \ldots, z_{N}\right)$. We assume that $A$ is irreducible, that is, not the union of two proper subvarieties, and that $\operatorname{dim} A=n$, that is, that the real (topological) dimension of $A$ is $2 n$. Griffiths' theorem asserts that there is a proper subvariety $B \subset A$, and a bounded domain $D \subset \mathbf{C}^{n}$ such that

$$
A-B=D / G
$$

where $G$ is a discrete fixed point free group of holomorphic automorphisms of $D$.

If $\operatorname{dim} A=1, A$ is an algebraic curve and we are in the Poincaré-KleinKoebe case. In this case $B$ is a finite set, which must contain all singular points of $A$, and $D$ may be chosen as a unit disc. If $\operatorname{dim} A>1$, however, $D$ will depend on $A$ and $B$. ( $B$ is not uniquely determined.)

The idea of the proof can be understood by considering a simple example. Let $A$ be the affine algebraic surface $(\operatorname{dim} A=2)$ in $C^{3}$ defined by the equation

$$
z_{1}^{2}-z_{2}\left(z_{2}-1\right)\left(z_{2}-2\right)\left(z_{2}-3\right)\left(z_{2}-z_{3}\right)=0
$$

and let $B$ be the intersection of $A$ with the algebraic (reducible) surface $z_{3}\left(z_{3}-1\right)\left(z_{3}-2\right)\left(z_{3}-3\right)=0$. Then, for $t \neq 0,1,2,3$ the intersection of $A-B$ with the plane $z_{3}=t$ is a Riemann surface $S(t)$ of type $(2,1)$; the single puncture corresponds to $z_{1}=z_{2}=\infty$. Thus $A-B$ is the set of pairs $(t, P)$ where $t \in \mathbf{C}-\{0,1,2,3\}=S_{0}$ and $P \in S(t)$.

Let $G_{0}$ be a torsion-free Fuchsian group with $U / G_{0}=S_{0}$ and let $\pi_{0}: U \rightarrow$ $S_{0}$ be the corresponding canonical projection. Let $X$ be the set of pairs $(\zeta, P)$ with $\zeta \in U$ and $P \in S\left(\pi_{0}(\zeta)\right)$. Then $X$ is a complex manifold and the mapping $(\zeta, P) \rightarrow\left(\pi_{0}(\zeta), P\right)$ is a holomorphic Galois (that is, unbounded, unramified and regular) covering of $A-B$ by $X$. 
Using the holomorphic dependence of $w^{\mu}$ on $\mu$ noted in $\$ 2$ and the theory of quasi-Fuchsian group sketched in $\S 6$, one can assign to each $\zeta \in U$ a torsion-free quasi-Fuchsian group $G_{\zeta}$ such that (i) $G_{i}$ is Fuchsian and $U / G_{i}=$ $S(\pi(i))$, (ii) $G_{\zeta}$ depends holomorphically on $\zeta$, in the sense that the generators of $G_{\zeta}$ may be chosen as holomorphic functions of $\zeta$, and (iii) if $\Delta_{1}(\zeta)$ and $\Delta_{2}(\zeta)$ are the components of the complement of the directed fixed Jordan curve $C(\zeta)$ of $G_{\zeta}$, lying to the left and to the right of that curve respectively, then

$$
\Delta_{1}(\zeta) / G_{\zeta}=S(\pi(\zeta)), \quad \Delta_{2}(\zeta) / G_{\zeta}=L / G_{i} .
$$

We denote the corresponding canonical projections of $\Delta_{1}(\zeta)$ onto $S(\pi(\zeta))$ by $\pi_{\xi}$.

Let $Y$ be the set of pairs $(\zeta, z)$ with $\zeta \in U, z \in \Delta_{1}(\zeta)$. Then $Y$ is a complex manifold and the mapping $(\zeta, z) \mapsto\left(\zeta, \pi_{\zeta}(z)\right)$ is a holomorphic Galois covering of $X$ by $Y$. But $Y$ is simply connected, hence it is a (holomorphic) universal covering space of $A-B$.

To obtain (18) it remains to show that the domain $Y \subset C^{2}$ is holomorphically equivalent to a bounded domain $D$. The argument involves a simple application of Koebe's one-quarter theorem and shall be omitted.

Examples of representations (18), and extensions to groups $G$ with fixed points, will be found in the recent thesis by G. Riera [59].

8. We proceed to state the second basic theorem-Teichmüller's theorem. The statement involves the concept of quadratic differentials. A (holomorphic) quadratic differential $\Phi$ on a Riemann surface $S$ is a holomorphic section of twice the canonical line bundle. This means that $\Phi$ assigns to every local parameter $z$ defined in a domain $\Delta \subset S$ a holomorphic function $\varphi(z)$, in such a way that $\varphi(z) d z^{2}$ is independent of the choice of the parameter; by abuse of language one writes $\Phi \mid \Delta=\varphi(z) d z^{2}$. With every quadratic differential $\Phi$ there is associated a density $|\Phi|$; locally $|\Phi|=$ $|\varphi(z)| d x d y$. A quadratic differential is called integrable if $\iint_{S}|\Phi|<\infty$, normalized if $\iint_{S}|\Phi|=1$. If $S=U / G, G$ a torsion-free Fuchsian group, every quadratic differential is represented as a holomorphic automorphic form of weight (-4), i.e., a holomorphic function $\varphi(z), z \in U$, satisfying

$$
\varphi(g(z)) g^{\prime}(z)^{2}=\varphi(z), \quad g \in G .
$$

If $\Phi \neq 0$, the curves along which $\Phi>0$ or $\Phi<0$ are called the horizontal and vertical trajectories of $\Phi$, respectively. They are, of course, mutually orthogonal. Near every $P \in S$ one can define a local parameter $\zeta$, called a natural parameter for $\Phi$, such that $\zeta=0$ at $P$ and

$$
\Phi=\left(\frac{r+2}{2}\right)^{2} \zeta^{r} d \zeta^{2} \text { near } P .
$$

The positive integer $r$, the order of $\Phi$ at $P$ is, clearly, uniquely determined; so is $\zeta$, except that it may be replaced by $\theta \zeta$ where $\theta^{r+2}=1$.

If $Q$ is a puncture on $S$ and $\Phi$ a quadratic differential which is integrable near $Q$ (and not identically zero), then $\Phi$ may have a simple pole at $Q$. In this case there is a (unique) natural local parameter $\zeta$ such that $\zeta=0$ at $Q$ and 


$$
\Phi=\frac{1}{4} \frac{d \zeta^{2}}{\zeta} \text { near } Q
$$

In Figure 1 we show the shape of horizontal trajectories of $\Phi$ near a point with $r=0,1,2$ and -1 .
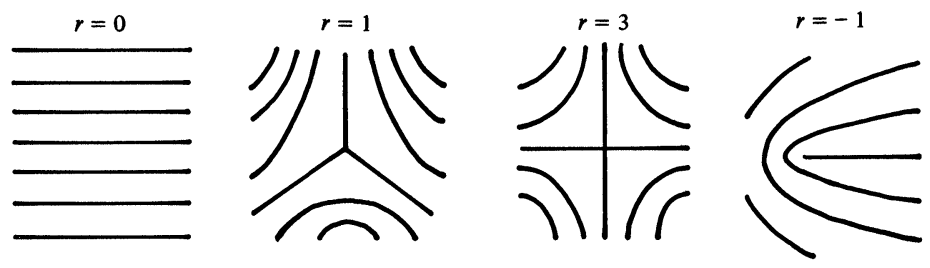

FIGURE 1

If $S$ is a Riemann surface of type $(p, n)$, then the integrable quadratic differentials on $S$ form a complex vector space of dimension $3 p-3+n$. (This follows from the Riemann-Roch theorem.)

Teichmüller's theorem ([64], [65], cf. also Ahlfors [1], Bers [8], Krushkal [41], and Hamilton [36]) deals with a variational problem. Let $S_{1}$ and $S_{2}$ be two Riemann surfaces of type $(p, n)$, with $2 p-2+n>0$, and $f$ a given orientation preserving homeomorphism of $S_{1}$ onto $S_{2}$. Among all quasiconformal mappings of $S_{1}$ onto $S_{2}$, homotopic to $f$, we want to find an extremal one, that is, one with the smallest dilatation.

(TeCHNICAL REMARK. The concepts of quasiconformality and of dilatation for mappings among Riemann surfaces are defined in terms of local parameters. This involves no difficulties, since the dilatation of a plane quasiconformal mapping $w$ is unchanged if $w$ is preceded or followed by a conformal mapping.)

Teichmüller's theorem asserts that (i) an extremal mapping $f_{0}$ always exists, (ii) is unique, and (iii) has a definite form.

Namely, if $K\left(f_{0}\right)>1$, that is, if $f_{0}$ is not conformal, then there exist on $S_{1}$ and on $S_{2}$ two uniquely determined normalized quadratic differentials $\Phi$ and $\Psi$, called the initial and terminal quadratic differentials, respectively, such that, for every $P \in S$,

$$
\operatorname{ord}_{P} \Phi=\operatorname{ord}_{f_{0}(P)} \Psi
$$

and, if $\operatorname{ord}_{P} \Phi=0$, then there are natural parameters: $z=x+i y$ for $\Phi$ at $P$ and $\zeta=\xi+i \eta$ for $\Psi$ at $f_{0}(P)$, such that near $P$ the mapping $f_{0}$ is given by

$$
\xi=K^{1 / 2} x, \quad \eta=K^{-1 / 2} y \quad \text { where } K=K\left(f_{0}\right) .
$$

In particular, $f_{0}$ takes the horizontal and vertical trajectories of $\Phi$ into those of $\Psi$.

Part (i) of the theorem is a direct consequence of compactness properties of quasiconformal mappings. Today there are several proofs for the characterization (iii). There is essentially only one proof of the uniqueness statement (ii), Teichmüller's ingenious extension of the length-area argument used by Grötzsch.

Remarks. (a) The variational problem leading to Teichmüller's theorem is an extension of a simpler problem first considered by Grötzsch. The great 
merit of recognizing that the quantity to be minimized is the supremum of the local dilatation rather than some average of the local dilatation is due to him.

(b) We have no time to report here on the important investigations by Jenkins and by Strebel on quadratic differentials with closed trajectories and by Hamilton, Reich, and Strebel on the extensions of Teichmüller's problem to mappings of domains and open Riemann surfaces with prescribed boundary correspondence. See [61], [63] for references.

9. The following variational problem is a rather natural extension of the Teichmüller problem, though actually I thought of it (and of the solution) only after learning of Thurston's beautiful theorem [66] on the classification of topological self-mappings of surfaces up to isotopy (or, which is the same in this case, up to homotopy).

Let $S$ be an oriented surface of type $(p, n)$, that is, homeomorphic to a compact surface of genus $p$ with $n$ distinct points removed. We assume that $2 p-2+n>0$. Also, let $f$ be an orientation preserving homeomorphism of $S$ onto itself. If $f^{\prime}: S \rightarrow S$ is isotopic to $f$ and if $\sigma$ is some conformal structure on $S$ (which makes $S$ into a Riemann surface $S_{\sigma}$ ), then it makes sense to measure the dilatation $K$ of $f^{\prime}$ with respect to $\sigma$; we write $K=K_{\sigma}\left(f^{\prime}\right)$. We want to find a conformal structure $\sigma_{0}$ on $S$ and a mapping $f_{0}$ isotopic to $f$ such that $K_{\sigma_{0}}\left(f_{0}\right)$ be as small as possible. If such a pair $\left(\sigma_{0}, f_{0}\right)$ exists, we call $\sigma_{0}$ an $f$-minimal conformal structure and we call $f_{0}$ an absolutely extremal selfmapping of the Riemann surface $S_{\sigma_{0}}$.

It is known, and easy to prove, that a conformal structure $\sigma_{0}$ on $S$ and a mapping $f_{0}$ isotopic to $f$ such that $K_{\sigma_{0}}\left(f_{0}\right)=1$, i.e., such that $f_{0}: S_{\sigma_{0}} \rightarrow S_{\sigma_{0}}$ is conformal, exist if and only if $f$ is isotopic to a mapping $f_{1}$ with $f_{1}^{m}=$ id for some $m>0$. If $f$ does not have this property, we call it essentially nonperiodic.

It can be shown that if $f$ is essentially nonperiodic, then we may, in treating the variational problem stated above, restrict ourselves to conformal structures $\sigma$ such that the Riemann surface $S_{\sigma}$ has $n$ punctures but no ideal boundary curves.

A mapping $f: S \rightarrow S$ will be called reducible if there is a mapping $f^{\prime}$ isotopic to $f$ and $r>0$ Jordan curves $C_{1}, \ldots, C_{r}$ on $S$, such that no $C_{j}$ is homotopic to a point or a puncture on $S$, or to a $C_{k}$ with $k \neq j$, and such that for each $j$ there is a $k$ with $f^{\prime}\left(C_{j}\right)=C_{k}$.

If $f$ is essentially nonperiodic, then an $f$-minimal conformal structure on $S$ exists if and only if $f$ is irreducible.

A proof of this statement, based on the direct method in the calculus of variations and on Teichmüller's theorem, will be found in the forthcoming paper [17]. The same paper contains a characterization of absolutely extremal mappings.

A nonconformal extremal self-mapping $f_{0}$ of a Riemann surface $S$ (of type $(p, n)$, with $2 p-2+n>0)$ onto itself is absolutely extremal if and only if its initial and terminal quadratic differentials coincide.

Assume this is so, and let $\Phi$ be the initial and terminal quadratic differential. The horizontal trajectories of $\Phi$ are the leaves of a foliation of $S$, with finitely many singularities, " $(r+2)$-pronged" singularities at points at which the order of $\Phi$ is $r \neq 0$ (cf. Figure 1 and note that $r=-1$ can happen only at the punctures of $S$ ). The vertical trajectories of $\Phi$ are the leaves of a 
transversal foliation. Both foliations are "measured", i.e., there is a consistent way of measuring the distance between two leaves, namely by the Riemannian conformal metric $d s=|\Phi|^{1 / 2}$. The mapping $f_{0}$ takes each of the two foliations into itself, and, by (19), multiplies the distance between vertical trajectories by the constant $K^{1 / 2}$ and that between horizontal trajectories by $K^{-1 / 2}$, where $K$ is the dilatation of $f$. A map with these properties is called by Thurston a pseudo-Anosov-diffeomorphism.

Thus we have proved, using quasiconformal mappings, one of Thurston's topological theorems. An essentially nonperiodic orientation preserving topological self-mapping of a surface $S$ (of the type considered) is either reducible or isotopic to a pseudo-Anosov-diffeomorphism but not both.

(The last statement follows from the detailed proof.)

10. An application of Teichmüller's theorem going back to Teichmüller himself is a new proof of the difficult part of a classical theorem by Fricke. To explain this theorem we must define the Teichmüller space $T_{p, n}$ (which, by the way, could have been called Fricke space) .

Let $S$ be an oriented surface of type $(p, n), 2 p-2+n>0$. A marked Riemann surface of type $(p, n)$ is an orientation preserving homeomorphism $f: S \rightarrow f(S)$ where $f(S)$ is a Riemann surface. Another marked Riemann surface $f_{1}: S \rightarrow f_{1}(S)$ is called equivalent to $f$ if $f_{1}=h \circ f \circ g$ where $g$ is isotopic to the identity and $h$ is conformal. The set of all equivalence classes [f] is called $T_{p, n}$. It is a complete metric space under the Teichmüller distance

$$
\delta\left(\left[f_{1}\right],\left[f_{2}\right]\right)=\frac{1}{2} \inf \log K\left(f^{\prime}\right), \quad f^{\prime} \text { homotopic to } f_{2} \circ f_{1}^{-1} \text {. }
$$

(One does not need Teichmüller's theorem to prove this.)

Every orientation preserving topological self-mapping $g$ of $S$ onto itself induces an isometry $g_{*}$ of $T_{p, n}$ which sends the equivalence class of $f: S \rightarrow$ $f(S)$ into that of $f \circ g: S \rightarrow f(S)$. These isometries form the modular group $\operatorname{Mod}_{p, n}$. It is clear that two points of $T_{p, n}$ are equivalent under $\operatorname{Mod}_{p, n}$ if and only if they can be represented by mappings of $S$ onto the same Riemann surface. It is equally clear that $g_{*} \in \operatorname{Mod}_{p, n}$ depends only on the homotopy class of $g$.

Fricke's theorem reads: The Teichmüller space $T_{p, n}$ is homeomorphic to $\mathbf{R}^{6 p-6+2 n}$; the modular group $\operatorname{Mod}_{p, n}$ acts properly discontinuously.

The difficult statement is the first one (see Fricke-Klein [30, pp. 284-394]; [31, pp. 285-310]; a modernized version of Fricke's argument will be found in Keen [38]).

In order to establish Fricke's statement about $T_{p, n}$ from Teichmüller's theorem we give the reference surface $S$, used in the definition of $T_{p, n}$, a conformal structure. Then every marked Riemann surface of type $(p, n)$ is equivalent to a unique extremal map $f: S \rightarrow f(S)$. This $f$ has some dilatation $K=e^{2 \rho} \geqslant 1$ and, if $\rho>0$, a (normalized) initial quadratic differential $\Phi$; if $\rho=0$, then $f=$ id. Conversely, every normalized quadratic differential $\Phi$ and every $\rho \geqslant 0$ determine an extremal map $f: S \rightarrow f(S)$. We may interpret $\Phi$ as a direction in $\mathbf{R}^{6 p-6+2 n}$ (since the real dimension of the space of quadratic differentials is $6 p-6+2 n)$ and $\rho$ as the distance from the origin. This gives a bijection between $\mathbf{R}^{6 p-6+2 n}$ and $T_{p, n}$, and it turns out that this bijection is bicontinuous. 
Fricke's theorem has been used by Earle and Eells to establish the cases $p=1$ and $p>1$ of their theorem, cf. $\S 4$. Another topological application of Fricke's theorem will be discussed presently.

11. The following theorem goes back to Nielsen:

(a) Let $g$ be an orientation preserving homeomorphism of a surface $S$ onto itself. If $g^{m}$ is homotopic to the identity, then $g$ is homotopic to a mapping $g_{0}$ such that $g_{0}^{m}=\mathrm{id}$. (Here $S$ is assumed to be oriented and of type $(p, n)$, $2 p-2+n>0$.)

The hypothesis of (a) implies that the modular transformation $g_{*} \in \operatorname{Mod}_{p, n}$ is periodic: $g_{*}^{m}=\mathrm{id}$. Assume that $g_{*}$ has a fixed point $\tau \in T_{p, n}$, and let $\tau$ be represented by $f: S \rightarrow f(S)$. Let us give to $S$ a conformal structure which makes $f$ into the identity. The condition $g_{*}(\tau)=\tau$ means that $g: S \rightarrow S$ is homotopic to a conformal map $g_{0}$. Since $\left(g_{0}^{m}\right)_{*}=g_{*}^{m}=$ id, $g_{0}^{m}$ is homotopic to the identity, and hence, being conformal, is the identity. This argument can be reversed and shows that (a) is equivalent to the following statement:

( $\left.a^{\prime}\right)$ If $g_{*} \in T_{p, n}$ and $g_{*}^{m}=$ id for some $m>0$, then $g_{*}$ has a fixed point.

If $m$ is the power of a prime, $\left(a^{\prime}\right)$ follows directly from Fricke's theorem and from the Paul Smith periodicity theorem. If not, one decomposes $m$ into its prime power factors and applies the Paul Smith theorem repeatedly, making use of the following auxiliary result: If $F$ is the set of points in $T_{p, n}$ fixed under an element of $\operatorname{Mod}_{p, n}$, then every component $F_{0}$ of $F$ can be identified with a $T_{p^{\prime}, n^{\prime}}$, and the action of the stabilizer of $F_{0}$ in $\operatorname{Mod}_{p, n}$ can be identified with the action of a subgroup of $\operatorname{Mod}_{p^{\prime}, n^{\prime}}$. This is so since the quotient of a Riemann surface by a finite group of conformal self-mappings is again a Riemann surface.

This elegant proof of (a) and (a') is due to Fenchel [29], cf. also Kravetz [40]. There are reasons to believe that $\left(a^{\prime}\right)$ can be replaced by the stronger result.

(b') Every finite subgroup of $\operatorname{Mod}_{p, n}$ has a fixed point.

This is equivalent to

(b) Let $\Gamma$ be a finitely generated group of orientation preserving topological self-mappings of $S$ and $\Gamma_{0}$ the group of homotopy classes of elements of $\Gamma$. If $\Gamma_{0}$ is finite, then the generators of $\Gamma$ are homotopic to generators of a finite group.

If $T_{p, n}$ were "of negative curvature in the sense of Busemann" (see Linch [47] for definitions), ( $\left.b^{\prime}\right)$ would follow, but Masur [53] showed it is not. On the other hand, the argument used above to prove $\left(a^{\prime}\right)$ also establishes $\left(b^{\prime}\right)$ for the case when the finite group in question is solvable. Other special cases of (b) have also been established.

(Thurston observed that his classification theorem yields a proof of (a) which is independent of Smith periodicity.)

12. I now would like to point out some connections between quasiconformal mappings, schlicht functions, and ordinary differential equations in the complex domain.

Recall that if $w(z)$ is a locally schlicht holomorphic (or meromorphic) function, its Schwarzian

$$
\varphi(z)=\{w, z\}=\frac{w^{\prime \prime \prime}(z)}{w^{\prime}(z)}-\frac{3}{2} \frac{w^{\prime \prime}(z)^{2}}{w^{\prime}(z)^{2}}
$$


is holomorphic. Conversly, every holomorphic function $\varphi(z)$ is a Schwarzian of a locally schlicht function

$$
w(z)=\eta_{1}(z) / \eta_{2}(z)
$$

where $\eta_{1}, \eta_{2}$ are two linearly independent solutions of the ordinary differential equation

$$
2 \eta^{\prime \prime}(z)+\varphi(z) \eta(z)=0 .
$$

Thus $w$ is determined by $\{w, z\}=\varphi$ uniquely, except that it may be replaced by $\alpha \circ w, \alpha$ a Möbius transformation.

It will be convenient to consider holomorphic functions $w(z)$ defined in the lower half-plane $L$. If $w$ is schlicht, it satisfies the Kraus-Nehari inequality [39], [56]:

$$
y^{2}|\varphi(z)| \leqslant \frac{3}{2} \quad(\varphi=\{w, z\})
$$

Nehari proved that if

$$
y^{2}|\varphi(z)| \leqslant \frac{1}{2} \quad(\varphi=\{w, z\})
$$

then $w$ is schlicht. (Both inequalities are sharp, Hille [37].) Hence it is reasonable to consider Schwarzians as elements of the complex Banach space B of holomorphic functions $\varphi(z), z \in L$, with norm

$$
\|\varphi\|=\sup \left|y^{2} \varphi(z)\right|<+\infty .
$$

The set $\mathbf{S} \subset \mathbf{B}$ of Schwarzians of schlicht functions is bounded and closed, but I do not know whether it is connected.

More accessible is the subset $T(1) \subset \mathbf{S}$ consisting of Schwarzians of functions admitting an extension to a quasiconformal self-mapping of $\hat{\mathbf{C}}=\mathbf{C}$ $\cup\{\infty\}$. The set $T(1)$ is called the universal Teichmüller space.

Every element of $T(1)$ can be written, by definition, as

$$
\varphi^{\mu}(z)=\left\{w^{\mu} \mid L, z\right\}
$$

where $\mu(z)$ is a measurable function with $\|\mu\|_{\infty}<1$ and $\mu \mid L=0$. Denote the set of all such functions by $\mathbf{M}$. For every $\mu \in \mathbf{M}$ we can form the function $w_{\mu}$. An easy but basic theorem (see, for instance, [10]) asserts that

$$
\varphi^{\mu_{1}}=\varphi^{\mu_{2}} \text { if and only if } w_{\mu_{1}}\left|\mathbf{R}=w_{\mu_{2}}\right| \mathbf{R} \text {. }
$$

For $\varphi \in T(1)$, inequality (20) can be sharpened to

$$
\left\|\varphi^{\mu}\right\| \leqslant \frac{3}{2}\|\mu\|_{\infty}
$$

(Kühnau [42], cf. Lehto [45]). On the other hand, if instead of (21) one has $\|\varphi\|<\frac{1}{2}$, then, according to Ahlfors and Weill [6], $\varphi \in T(1)$ and

$$
\varphi=\varphi^{\mu} \quad \text { where } \mu(z)=-2 y^{2} \varphi(\bar{z}) \text { for } z \in U .
$$

If $\varphi=\{w, z\} \in T(1)$, then $w(L)$ is, of course, a Jordan domain and we may assume, without loss of generality that its boundary curve $C$ contains $z=\infty$. Ahlfors [3] proved that if $a, b, c$ are any three finite points on $C$ following each other in this order, then

$$
\left|\frac{b-a}{c-a}\right| \leqslant M
$$


for some constant $M$, and that, conversely the existence of such a constant is sufficient in order that the conformal map of $L$ onto the domain bounded by $C$ admit a quasiconformal extension.

The set $T(1)$ turns out to be a domain (Ahlfors [3]) which is contractible (Earle and Eells [26]), homogeneous (Bers [12], [13]), and holomorphically convex (Bers and Ehrenpreis [18]). It would be pleasant if $\mathbf{S}$ were the closure of $T(1)$, but whether this is so is an open question. However, recently Gehring [33] proved that $T(1)$ is the interior of $\mathbf{S}$.

13. If $G$ is a discrete group of real Möbius transformations, i.e., a Fuchsian group, we denote by $\mathbf{B}(G)$ the closed linear subspace of $\mathbf{B}$ consisting of those $\varphi$ which satisfy the functional equation of quadratic differentials

$$
\varphi(g(z)) g^{\prime}(z)^{2}=\varphi(z), \quad g \in G .
$$

If $G$ is finitely generated, $T(1) \cap \mathbf{B}(G)$ is known to be connected; in all cases we call the component of $T(1) \cap \mathbf{B}(G)$ containing the origin the Teichmüller space of $G$, and we denote it by $T(G)$.

A calculation shows that $\varphi^{\mu}, \mu \in \mathbf{M}$, belongs to $T(G)$ if and only if

$$
\mu(g(z)) \overline{g^{\prime}(z)} / g^{\prime}(z)=\mu(z), \quad g \in G .
$$

This requirement is equivalent to the condition that $G^{\mu}=w^{\mu} G\left(w^{\mu}\right)^{-1}$ be a quasi-Fuchsian group and that $G_{\mu}=w_{\mu} G w_{\mu}^{-1}$ be a Fuchsian group.

Clearly, $\operatorname{dim} T(G)=\operatorname{dim} \mathbf{B}(G)$. This dimension is finite if and only if $G$ is

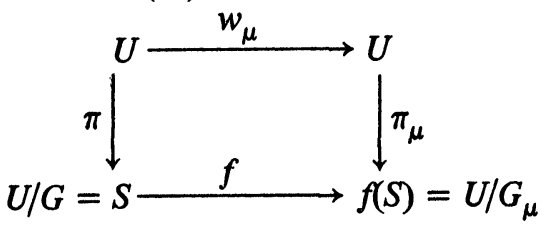

finitely generated and does not act properly discontinuously on any real interval, and if and only if the Riemann surface $U / G$ is compact, except perhaps for finitely many punctures.

If $\operatorname{dim} \mathbf{B}(G)<\infty$ and $\varphi \in \mathbf{B}(G)$, then $\varphi \in T(G)$ if and only if $\varphi$ is the Schwarzian of a conformal mapping of $L$ onto some Jordan domains. In other words, in this case the Ahlfors condition (23) is automatically satisfied.

If $\operatorname{dim} \mathbf{B}(G)<\infty$ and $G$ is torsion free, then $T(G)$ can be identified with $T_{p, n},(p, n)$ being the type of $U / G$ ([11] and [12], [13], [26], [27] for more general groups). For if $\varphi \in T(G)$, then $\varphi=\varphi^{\mu}, \mu \in \mathbf{M}$ where $\mu$ satisfies (24) and $w_{\mu} G w_{\mu}^{-1}=G_{\mu}$ is Fuchsian. This implies a commutative diagram where $\pi$ and $\pi_{\mu}$ are canonical projections. The map $f$ represents an element of $T_{p, n}$ (cf. §10), and all elements of $T_{p, n}$ can be so obtained. It follows from (22) that the point in $T_{p, n}$ depends only on $\varphi$ and not on the $\mu \in M$ occurring in the above diagram. The resulting bijection $T(G) \rightarrow T_{p, n}$ turns out to be a homeomorphism.

This method of representing $T_{p, n}$ as a bounded domain in $\mathbf{C}^{3 p-3+n}$ (note that $\operatorname{dim} \mathbf{B}(G)=3 p-3+n)$ is one way of giving $T_{p, n}$ a complex structure. There are several others, and they are all equivalent. It is at this point that the deeper theory of Teichmüller spaces begins. But we cannot pursue these matters here.

(If $\operatorname{dim} \mathbf{B}(G)<\infty$ and $G$ has elements of finite order, $T(G)$ is 
holomorphically equivalent to $T_{p, n+r}$ where $(p, n)$ is again the type of $U / G$ and $r$ is the number of nonconjugate maximal finite cyclic subgroups of $G$. The original proofs of this statement, by Bers and Greenberg [19] and by Marden [49] were complicated; Kra gave a very short proof, based on Teichmüller's theorem, see [27].)

14. The embedding of $T_{p, n}$ into $\mathrm{C}^{3 p-3+n}$ constructed in $\$ 13$ may be called semicanonical. It is not fully canonical since it depends on the choice of a torsion-free Fuchsian group $G$, with $U / G$ of type $(p, n)$, and also, which is less significant, on the choice of a basis in $\mathbf{B}(G)$.

Little is known about the actual shape of $T_{p, n}$ in $\mathbf{C}^{3 p-3+n}$, except for the negative information contained in the fact that $T_{p, n}$ is not a homogeneous domain if $3 p-3+n>1$. This result is due to Royden [60].

Finding the semicanonical embedding amounts to solving an accessory parameters problem for a second order ordinary linear differential equation. Already the simplest case is difficult.

Let $\wp(z)$ denote the Weierstrass $\wp$-function belonging to the primitive periods $(1, \tau)$ where $\tau \in U$. We consider Lamés equation for $n=-\frac{1}{2}$, that is, the equation

$$
\eta^{\prime \prime}(z)+\left[\frac{1}{4} \wp(z)+\lambda\right] \eta(z)=0,
$$

and denote two linearly independent solutions by $\eta_{1}$ and $\eta_{2}$. Classical uniformization theory asserts that for each $\tau$ there is precisely one $\lambda$ such that

$$
\frac{\eta_{1}}{\eta_{2}}(\mathbf{C}-\text { the periods of } \wp)=\left\{\begin{array}{l}
\text { a simply covered disc } \\
\text { or half plane. }
\end{array}\right.
$$

This is so since condition (25) is necessary and sufficient in order that the mapping of the disc or half-plane inverse to $\eta_{1} / \eta_{2}$, followed by the mapping of $\left(\mathbf{C}\right.$ - the periods) by the pair $\left(\wp(z), \wp^{\prime}(z)\right)$ be a universal covering of $S_{j(\tau)}$, a Riemann surface of type $(1,1)$ belonging to $j(\tau)$.

However, the actual value of $\lambda$ has been found in only a few cases.

Now we ask: for which values of $\lambda$ is

$$
\frac{\eta_{1}}{\eta_{2}}(\mathbf{C}-\text { the periods of } \wp)=\left\{\begin{array}{l}
\text { a simply covered } \\
\text { Jordan domain? }
\end{array}\right.
$$

The theory exposed in $\S 13$ gives a qualitative answer: for all $\lambda$ in some simply connected bounded domain $\Delta$, depending only on $j(t)$. This is so since it can be shown that the set of $\lambda$ satisfying (26) is the semicanonical image of $T_{1,1}$ in $\mathbf{C}$, determined by a Fuchsian group $G$ with $U / G=S_{j(\tau)}$.

It would be important, in view of some questions concerning Kleinian groups, to know what $\Delta$ is. In particular is $\Delta$ a disc for some, or for all, values of $\tau$ ? This concrete problem may be a good place to end.

\section{REFERENCES}

This list is by no means exhaustive

1. L. V. Ahlfors, On quasiconformal mappings, J. Analyse Math. 3 (1954), 1-58. MR 16, 348.

2. Conformality with respect to Riemannian matrices, Ann. Acad. Sci. Fenn. Ser. A. I., no. 206 (1955), 1-22. MR 17, 657.

3. Q Quasiconformal reflections, Acta. Math. 109 (1963), 291-301. MR 27 \#4921. 
4. __ Lectures on quasiconformal mappings, Van Nostrand, Princeton, N.J., 1966. MR 34 \#336.

5. L. V. Ahlfors and L. Bers, Riemann's mapping theorem for variable metrics, Ann. of Math. (2) 72 (1960), 385-404.

6. L. V. Ahlfors and G. Weill, A uniqueness theorem for Beltrami equations, Proc. Amer. Math.

Soc. 13 (1962), 975-978. MR 26 \#6393.

7. L. Bers, Existence and uniqueness of a subsonic flow past a given profile, Comm. Pure Appl. Math. 7 (1954), 441-504. MR 16, 417.

8. Q Quasiconformal mappings and Teichmüller's theorem, Analytic Functions, Princeton Univ. Press, Princeton, N.J., 1960, 89-119. MR 22 \#5716.

9. __ Simultaneous uniformization, Bull. Amer. Math. Soc. 66 (1960), 94-97. MR 22 \#2694.

10. , Uniformization by Beltrami equations, Comm. Pure Appl. Math. 14 (1961), 215-228. MR 24 \# A2022.

11. Correction to "Spaces of Riemann surfaces as bounded domains", Bull. Amer. Math. Soc. 67 (1961), 465-466. MR 24 \# A826.

12. __ Automorphic forms and general Teichmüller spaces, Proc. Conf. Complex Analysis (Minneapolis, 1964), Springer-Verlag, Berlin, 1965, pp. 109-113. MR 31 \#2400.

13. __ On moduli of Riemann surfaces, Lectures at the Forschunginstitut für Mathematik,

Eidgenössische Technische Hochschule (Zurich, Summer 1964), mimeographed.

14. _ Automorphic forms and Poincaré series for infinitely generated Fuchsian groups, Amer. J. Math. 87 (1965), 196-214. MR 30 \#4937.

15. __ Uniformization moduli and Kleinian groups, Bull. London Math. Soc. 4 (1972), 257-300. MR 50 \# 595.

16. __ On Hilbert's 22nd problem, Proc. Sympos. Pure Math. vol. 28, Amer. Math. Soc., Providence, R.I., 1976. 559-609.

17. __ An extremal problem for quasiconformal mappings and a theorem by Thurston, Acta Math. (to appear).

18. L. Bers and L. Ehrenpreis, Holomorphic convexity of Teichmüller spaces, Bull. Amer. Math. Soc. 70 (1964), 761-764. MR 29 \#6065.

19. L. Bers and L. Greenberg, Isomorphisms between Teichmüller spaces, Advances in the Theory of Riemann Surfaces, Ann. of Math. Studies, No. 66, Princeton Univ. Press, Princeton, N.J., 1971, pp. 53-79. MR 44 \#6951.

20. L. Bers and I. Kra (Editors), A crash course on Kleinian groups, Lecture Notes in Math., vol. 400, Springer-Verlag, Berlin and New York, 1974.

21. L. Bers and L. Nirenberg, On a representation theorem for linear elliptic systems with discontinuous coefficients and its applications, Convegno Internazionale sulle Equazioni Lineari alle Derivati Parziali (Trieste, 1954), Edizioni Cremonese, Roma, 1955, pp. 111-140. MR 17, 974.

22. __ On linear and non-linear elliptic boundary value problems in the plane, Convegno Internazionale sulle Equazioni Lineari alle Derivati Parziali (Trieste, 1954). Edizioni Cremonese, Roma, 1955, pp. 141-167. MR 17, 974.

23. C. J. Earle, Reduced Teichmüller spaces, Trans. Amer. Math. Soc. 126 (1967), 54-63. MR 34 \#4481.

24. (1964), 699-701. MR 30 \#3207.

25. C. J. Earle and J. Eells, A fibre bundle description of Teichmüller theory, J. Differential Geometry 3 (1969), 19-43. MR 43 \#2737A.

26. __ On the differential geometry of Teichmüller spaces, J. Analyse Math. 19 (1967), 35-52. MR 36 \#3975.

27. C. J. Earle and I. Kra, On holomorphic mappings between Teichmüller spaces, edited by L. V. Ahlfors et al., Contributions to Analysis, Academic Press, New York, 1974, pp. 107-124.

28. C. J. Earle and A. Schatz, Teichmüller theory for surfaces with boundary, J. Differential Geometry 4 (1970), 169-185. MR 43 \#2737B.

29. W. Fenchel, Estensioni di gruppi discontinui e trasformazioni periodiche delle superficie, Atti

Acad. Naz. Lincei Rend. Cl. Sci. Fis. Mat. Nat. (8) 5 (1948), 326-329. MR 10, 558.

30. R. Fricke and F. Klein, Vorlesungen über die Theorie der automorphen Funktionen. I, Teubner, Leipzig, 1926. 
31. , Vorlesungen über die Theorie der automorphen Funktionen. II, Teubner, Leipzig, 1926.

32. F. W. Gehring, Some metric properties of quasiconformal mappings, Proc. Internat. Congr. Math. (Vancouver, 1974), vol. 2, Canad. Math. Congress, Montreal, 1975, pp. 203-206.

33. Univalent functions and Schwarzian derivatives, Comment. Math. Helv. (to appear).

34. A. Gramain, Le type d'homotopie du groupe des diffeomorphismes d'une surface, Ann. Sci. École Norm. Sup. (4) 6 (1973), 53-56. MR 48 \#116.

35. P. A. Griffiths, Complex analytic properties of certain Zariski open sets on algebraic varieties, Ann. of Math. (2) 94 (1971), 21-51. MR 46 \#9385.

36. R. S. Hamilton, Extremal quasiconformal mappings with prescribed boundary values, Trans. Amer. Math. Soc. 138 (1969), 399-406. MR 39 \# 7093.

37. E. Hille, Remarks on a paper by Zeev Nehari, Bull. Amer. Math. Soc. 55 (1949), 552-553. MR 10, 697.

38. L. Keen, On Fricke moduli, Advances in the Theory of Riemann Surfaces, Ann. of Math. Studies, No. 66, Princeton Univ. Press, Princeton, N.J., 1971, pp. 205-224. MR 44 \#5450.

39. W. Kraus, Über den Zusammenhang einiger Characteristiken eines einfach zurammenhängenden Bereiches mit Kreisabbildungen, Mitt. Math. Sem. Giessen 21 (1932), 1-28.

40. S. Kravetz, On the geometry of Teichmüller spaces and the structure of their modular groups, Ann. Acad. Sci. Fenn. Ser. A.I 278 (1959), 1-35. MR 26 \#6402.

41. S. L. Krushkal, On a Teichmüller theorem on extremal quasi-conformal mappings, Sibirsk Mat. Ż. 8 (1967), 313-332. (Russian) MR 35 \#5610.

42. R. Kühnau, Über die analytische Darstellung von Abbildungsfunktionen, insbesondere der Extremalfunktionen der Theorie der konformen Abbildung, J. Reine Angew Math. 228 (1967), 93-132. MR 37 \#394.

43. __ Wertannahmeprobleme bei quasikonformen Abbildungen mit ortsabhängiger Dilatatiousbeschränkung, Math. Nachr. 40 (1969), 1-11. MR 40 \#2853.

44. H. P. Künzi, Quasikonforme Abbildungen, Ergebnisse der Mathematik, vol. 26, SpringerVerlag, Berlin-Göttingen-Heidelberg, 1960.

45. O. Lehto, Schlicht functions with a quasiconformal extension, Ann. Acad. Sci. Fenn. Ser A.I 500 (1971). MR 45 \#3692.

46. O. Lehto and K. I. Virtanen, Quasikonforme Abbildungen, Springer-Verlag, Berlin and New York, 1965. MR 32 \# 5872.

47. M. R. Linch, On metrics in Teichmüller spaces, Ph.D. Thesis, Columbia University, 1971.

48. C. Loewner, On the conformal capacity in space, J. Math. Mech. 8 (1959), 411-414. MR 21 $\# 3538$.

49. A. Marden, On homotopic mappings of Riemann surfaces, Ann. of Math. (2) 90 (1969), 1-8. MR 40 \#2849.

50. B. Maskit, Classification of Kleinian groups, Proc. Internat. Congr. Math. (Vancouver, 1974), vol. 2, Canad. Math. Congr., Montreal, 1975, pp. 213-216.

51. _ Classification of Kleinian groups. I, Acta Math. 135 (1975), 249-270.

52. __ Classification of Kleinian groups. II, Acta Math. (to appear).

53. H. Masur, On a class of geodesics in Teichmüller space, Ann. of Math. (2) 102 (1975), 205-221.

54. C. B. Morrey, On the solutions of quasi-linear elliptic partial differential equations, Trans. Amer. Math. Soc. 43 (1938), 126-166.

55. G. D. Mostow, Quasiconformal mappings in n-space and rigidity of hyperbolic space forms, Inst. Hautes Etudes Sci. Publ. Math. No. 34 (1968), 53-104. MR 38 \# 4679.

56. Z. Nehari, The Schwarzian derivative and schlicht functions, Bull. Amer. Math. Soc. 55 (1949), 545-551. MR 10, 696.

57. L. Nirenberg, On non-linear elliptic partial differential equations and Hölder continuity, Comm. Pure Appl. Math. 6 (1953), 103-156; Appendum, p. 395. MR 16, 367.

58. _ On a generalization of quasiconformal mappings and its applications to elliptic partial differential equations, Contributions to the Theory of Partial Differential Equations, edited by $\mathbf{L}$. Bers et al., Ann. of Math. Studies No. 33, Princeton Univ. Press, Princeton, N.J., 1954, pp. 95-100. MR 16, 592.

59. G. Riera, Subdirect products of Fuchsian groups and uniformization, Duke Math. J. 44 (1977), 291-304. 
60. H. L. Royden, Automorphisms and isometries of Teichmüller spaces, Advances in the Theory of Riemann Surfaces, Princeton Univ. Press, Princeton, N.J., 1971, pp. 369-383. MR 44 \# 5452.

61. E. Reich and K. Strebel, Extremal quasiconformal mappings with given boundary values, Contributions to Analysis, edited by Ahlfors et al., Academic Press, New York, 1974, pp. 375-391. MR 50 \#13511.

62. S. Smale, Diffeomorphisms of the 2-sphere, Proc. Amer. Math. Soc. 10 (1959), 621-626. MR 22 \#3004.

63. K. Strebel, On quadratic differentials and extremal quasi-conformal mappings, Proc. Internat. Congr. Math. (Vancouver, 1974), vol. 2, Canad. Math. Congr., Montreal, 1975, pp. 223-227.

64. O. Teichmüller, Extremale quasikonforme Abbildungen und quadratische Differentiale, Abh. Preuss. Akad. Wis. Math.-Nat. Kl. 22 (1939).

65. __ Bestimmung der extremalen quasikonformen Abbildung bei geschlossenen orientierten Riemannschen Flächen, Abh. Preuss. Akad. Wis. Math.-Mat. Kl. 4 (1943). MR 8, 202.

66. W. P. Thurston, On the geometry and dynamics of diffeomorphisms of surface. I (to appear).

67. J. Väisälä, Lectures on n-dimensional quasiconformal mappings, Lecture Notes in Math., vol. 229, Springer-Verlag, Berlin and New York, 1971.

Department of Mathematics, Columbia University, New York, New York 10027 